\title{
Heterotic M-theory from the clockwork perspective
}

\author{
Sang Hui Im, ${ }^{a}$ Hans Peter Nilles ${ }^{b, c}$ and Marek Olechowski ${ }^{d}$ \\ ${ }^{a}$ Department of Physics, Pusan National University, \\ Busan 46241, Korea \\ ${ }^{b}$ Bethe Center for Theoretical Physics and Physikalisches Institut der Universität Bonn, \\ Nussallee 12, 53115 Bonn, Germany \\ ${ }^{c}$ Arnold Sommerfeld Center for Theoretical Physics, Ludwig-Maximilians-Universität München, \\ Theresienstraße 37, 80333 Munich, Germany \\ ${ }^{d}$ Institute of Theoretical Physics, Faculty of Physics, University of Warsaw, \\ ul. Pasteura 5, 02-093 Warsaw, Poland \\ E-mail: imsanghui@pusan.ac.kr, nilles@th.physik.uni-bonn.de, \\ Marek.Olechowski@fuw.edu.pl
}

ABSTRACT: Compactifications of heterotic M-theory are shown to provide solutions to the weak- and axion-scale hierarchy problems as a consequence of warped large extra dimensions. They allow a description that is reminiscent of the so-called continuous clockwork mechanism. The models constructed here cover a new region of clockwork parameter space and exhibit unexplored spectra and couplings of Kaluza-Klein modes. Previously discussed models are outside this region of parameter space and do seem to require an ultraviolet completion other than that of perturbative higher dimensional $D=10,11$ string- or M-theory. A 5D-supergravity description can be given for all explicitly known continuous clockwork models. The various classes of models can be distinguished through the different roles played by vector multiplets and the universal hypermultiplet in 5D-supergravity.

KeYwords: M-Theory, Supergravity Models, Superstrings and Heterotic Strings, Large Extra Dimensions

ArXiv ePrint: 1811.11838 


\section{Contents}

1 Introduction 1

2 General Linear Dilaton (GLD) in a nutshell 3

3 Hořava-Witten model (minimal heterotic M-theory) 6

4 Masses and couplings of Kaluza-Klein (KK) states 10

5 Heterotic M-theory with vector multiplets 12

6 Can we avoid the heterotic M-theory bound? 15

$\begin{array}{lll}7 & \text { Conclusions and outlook } & 17\end{array}$

\section{Introduction}

Understanding the origin of small couplings and large hierarchies of scales is a major challenge in theoretical physics model building. Various mechanisms have been explored, some based on a slight breakdown of custodial symmetries (as e.g. supersymmetry), others on peculiar properties of extra dimensions. Examples for the latter include large extra dimensions (LED) [1], warped extra dimensions (RS) [2] or the so-called linear dilaton (LD) model [3]. A comprehensive discussion can be formulated in the formalism of the general continuum clockwork mechanism (GCCW) as described in ref. [4].

In the present paper we report on investigations in the framework of heterotic M-theory [5] that makes connection to the GCCW and extends the mechanism in a nontrivial way that has not yet been explored previously. It also gives a consistent UV-completion of specific examples of the clockwork mechanism in string theory. These main results of our work will be explained in sections 3 through 5 of the paper.

Let us first give an introduction to the clockwork mechanism and the GCCW. The clockwork scheme can be viewed as a generalisation of the aligned axion mechanism $[6,7]$ originally proposed in the framework of high scale natural inflation. Its generalisation to the multi-axion case [8] has an interesting application beyond the inflationary picture for the scale of the QCD axion [9]. It is also well suited for the discussion of the so-called relaxion mechanism [10] as discussed in ref. [11] and [12]. ${ }^{1}$ A multi-axion picture with a large number of axions can be connected to schemes of deconstructions of extra dimensions along the lines of refs. [13, 14] with a discrete number of sites: the discrete clockwork (DCW). The transition to a continuous clockwork mechanism (CCW) was suggested in

\footnotetext{
${ }^{1}$ The name clockwork was first suggested in ref. [12].
} 
ref. [15]. Some specific properties of the DCW are lost in the generalisation to a CCW and this leads to some ambiguities in its definition and interpretation [16, 17]. A comprehensive description of the general picture (GCCW) is given in ref. [4] on which we base our present discussion. Various applications of the clockwork mechanism have been given in [18-55].

In this paper, our main focus will be on a subset of the GCCW known as the general linear dilaton model (GLD) [4]. It is described by two continuous parameters and it includes the well-known cases such as LED, RS and LD, but there are many more possibilities. The goal of the present paper is two-fold: first to explore the spectrum of GLD models beyond the examples known up to now and then provide a consistent ultraviolet (UV) completion within the framework of string theory, if possible.

In this paper we report on progress in both directions:

- we have found new solutions in the framework of heterotic M-theory,

- these differ decisively from previously discussed solutions as they exhibit a new structure for the Kaluza-Klein (KK) spectrum and couplings,

- we discuss the role of the universal hypermultiplet of compactified string theory for the properties for the UV-completion of GLD models and derive a bound on the parameters valid for the models obtained in the framework of heterotic M-theory,

- previously discussed models are shown to be mostly outside this bound and might face difficulties for a UV-completion in perturbative higher-dimensional $(D=10,11)$ string theory,

- we provide a 5D-supergravity description for all known models and discuss the different roles of vector- and hypermultiplets in 5D.

The paper will be structured as follows. In section 2 we shall give an introduction to the GLD models. We describe the appearance of the two basic parameters relevant for the creation of hierarchical scales and discuss their phenomenological consequences for the spectrum of the Kaluza-Klein modes and the hierarchies of couplings. We reproduce the results for the previously explored special cases LED, RS and LD. Only for these three discrete choices of parameters do we have a bottom-up construction of GLD models. The remaining part of parameter space still needs explicit realisations of these yet unexplored KK spectra and couplings. It remains an open question whether more examples can be realised in quantum field theory and string theory.

A (partial) answer will be given in sections 3 and 4 . Here we consider compactifications of heterotic M-theory that could explain the hierarchy between the Planck scale and hierarchically smaller scales as e.g. the electroweak scale or the scale of the invisible axion (which require special choices of parameters that do not coincide with the so-called standard embedding). This allows a connection towards the clockwork mechanism and provides new realisations of GLD models with phenomenological properties that differ qualitatively from the previous constructions (LED, RS and LD). As we have a consistent UV-completion we can give an explicit discussion in the framework of supergravity on a 5-dimensional (5D) 
manifold with the 5-th dimension being an interval. We stress the crucial role of the 5D universal hypermultiplet. Models with additional 5D vector multiplets are presented as well in section 5 .

In section 6 we shall discuss the clockwork mechanism from the 5D perspective and make connection to previous work that realises the linear dilaton model (LD) within 5Dsupergravity [56, 57]. The other known bottom-up constructions (among them RS) can be embedded in this scheme. From the 5D-supergravity perspective here the 5D vector multiplets play a crucial role, while the universal hypermultiplet is removed. Such a situation is impossible in heterotic M-theory. It remains an open question whether it can be embedded in any pertrubative higher dimensional $(D=10,11)$ string theory. Section 7 contains our conclusions and outlook towards a complete classification of GLD models with a consistent UV-completion.

\section{General Linear Dilaton (GLD) in a nutshell}

In this section, we will give a brief summary of the continuum clockwork and GLD model. The scalar clockwork action can be written as [15]

$$
\int \mathrm{d}^{4} x\left[\sum_{i=0}^{N} \frac{1}{2}\left(\partial_{\mu} \phi_{i}\right)^{2}+\sum_{i=0}^{N-1} \frac{1}{2} m_{i}^{2}\left(\phi_{i+1}-q \phi_{i}\right)^{2}\right],
$$

where we allow the clockwork gear mass parameter $m_{i}$ can depend on the site $i$. The continuum limit of the action is obtained by taking $N \rightarrow \infty$ and introducing 5 -th continuous coordinate, $y$, with

$$
\begin{aligned}
\sum_{i} & \rightarrow \frac{1}{\Delta r} \int_{0}^{\pi R} \mathrm{~d} y, & m_{i} & \rightarrow \frac{m(y)}{\Delta r}, \\
\phi_{i}(x) & \rightarrow \Phi(x, y) \Delta r^{1 / 2}, & \phi_{i+1}-\phi_{i} & \rightarrow \partial_{y} \Phi(x, y) \Delta r^{3 / 2},
\end{aligned}
$$

where $\Delta r \equiv \pi R / N$ is the lattice spacing with finite $R$. Here we introduce a dimensionless function $m(y)$ to parameterize the site-dependent mass parameter $m_{i}$. The resultant continuum clockwork action is

$$
\int \mathrm{d}^{5} x\left[\frac{1}{2}\left(\partial_{\mu} \Phi\right)^{2}+\frac{1}{2} m^{2}(y)\left(\partial_{y} \Phi-k \Phi\right)^{2}\right] .
$$

As we redefine the field $\Phi \rightarrow \Phi e^{k y}$, the action can be rewritten as

$$
\int \mathrm{d}^{5} x e^{2 k y}\left[\frac{1}{2}\left(\partial_{\mu} \Phi\right)^{2}+\frac{1}{2} m^{2}(y)\left(\partial_{y} \Phi\right)^{2}\right] .
$$

This action can be obtained from 5D diffeomorphism invariant lagrangian when the metric is replaced by a certain background value:

$$
\int \mathrm{d}^{5} x \sqrt{-g} \frac{1}{2} g^{\alpha \beta} \partial_{\alpha} \Phi \partial_{\beta} \Phi
$$


with

$$
\mathrm{d} s^{2}=e^{\frac{4}{3} k y} m^{\frac{2}{3}}(y)\left(\mathrm{d} x^{2}+m^{-2}(y) \mathrm{d} y^{2}\right) .
$$

Throughout this section, we will use $\alpha, \beta, \cdots=0, \ldots, 3,5$ while $\mu, \nu, \cdots=0, \ldots, 3$. As for the clockwork gear mass function $m(y)$, let us consider a simple exponential profile $m(y)=e^{p y}$. Then the corresponding background geometry is

$$
\mathrm{d} s^{2}=e^{\frac{4}{3} k y} e^{\frac{2}{3} p y}\left(\mathrm{~d} x^{2}+e^{-2 p y} \mathrm{~d} y^{2}\right) \equiv e^{2 k_{1} y} \mathrm{~d} x^{2}+e^{2 k_{2} y} \mathrm{~d} y^{2},
$$

where

$$
k=k_{1}+\frac{1}{2} k_{2}, \quad p=k_{1}-k_{2} .
$$

From the continuum clockwork action (2.3), it is clear that $k$ is responsible for generating coupling hierarchies, while $p$ controls the clockwork gear masses (KK masses) via the relation $m(y)=e^{p y}$.

The above background geometry can be generated by GLD proposed in [4]. The model is defined as the $5 \mathrm{D}$ dilaton-gravity action with the specific form of dilaton potential:

$$
\begin{aligned}
\mathcal{S}=M_{5}^{3} \int \mathrm{d}^{5} x \sqrt{-g}\left(\frac{1}{2} \mathcal{R}_{5}-\right. & \frac{1}{2} \partial_{\alpha} S \partial^{\alpha} S-\Lambda_{b} e^{-(2 \hat{c} / \sqrt{3}) S} \\
& \left.\quad-e^{-(\hat{c} / \sqrt{3}) S}\left[\Lambda_{0} \frac{\delta(y)}{\sqrt{g_{55}}}+\Lambda_{\pi} \frac{\delta(y-\pi R)}{\sqrt{g_{55}}}\right]\right),
\end{aligned}
$$

where the 5 -th dimension $y$ is compactified on an orbifold $S_{1} / \mathbb{Z}_{2}$ with the fixed points at $y=\{0, \pi R\}, M_{5}$ is the 5D Planck mass, $\left(\Lambda_{b}, \Lambda_{0}, \Lambda_{\pi}\right)$ are constants, and $\hat{c}$ is an arbitrary real parameter. ${ }^{2}$ If the potentials at the fixed points satisfy the following relation

$$
-\Lambda_{0}=\Lambda_{\pi}= \pm 6 \sqrt{\frac{2}{3}\left(\frac{\Lambda_{b}}{\hat{c}^{2}-4}\right)},
$$

it can be shown that there exists a $4 \mathrm{D}$ Minkowski background solution. ${ }^{3}$ Thus, we are left with two free parameters, $\hat{c}$ and $\Lambda_{b}$, in (2.9). The consequent background solution turns out to be just (2.7):

$$
\mathrm{d} s^{2}=e^{2 k_{1} y} \mathrm{~d} x^{2}+e^{2 k_{2} y} \mathrm{~d} y^{2},
$$

where $k_{1}$ and $k_{2}$ are determined in terms of $\hat{c}$ and $\Lambda_{b}$ :

$$
k_{1}= \pm \sqrt{\frac{2}{3}\left(\frac{\Lambda_{b}}{\hat{c}^{2}-4}\right)}, \quad k_{2}=\hat{c}^{2} k_{1} .
$$

Furthermore, the dilaton field is shown to have a linear dilaton background,

$$
(\hat{c} / \sqrt{3}) S=k_{2} y
$$

${ }^{2} \hat{c}=\sqrt{3} c$ where $c$ was introduced in [4].

${ }^{3}$ For a discussion of inflationary $4 \mathrm{D}$ solutions we refer to [20, 21]. 


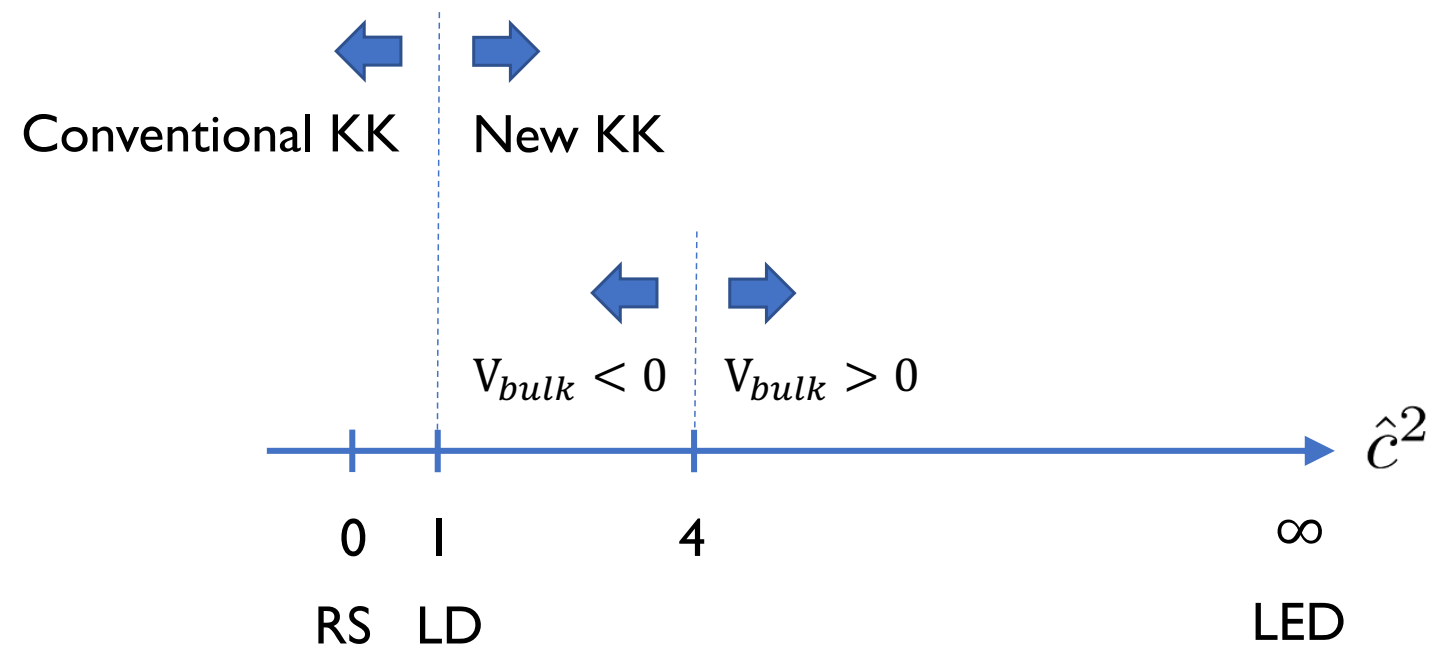

Figure 1. Critical points of $\hat{c}^{2}$.

From the GLD point of view, therefore, we can take $\left(\hat{c}, \Lambda_{b}\right)$ as two fundamental parameters to determine the properties of the clockwork instead of $(k, p)$ or $\left(k_{1}, k_{2}\right)$. From the solution (2.11), one can notice that there are critical values for $\hat{c}^{2}$. For $k_{1}$ to be real, the bulk potential has to be negative $\left(\Lambda_{b}<0\right)$ for $\hat{c}^{2}<4$ while positive $\left(\Lambda_{b}>0\right)$ for $\hat{c}^{2}>4$. If $\hat{c}^{2}=4$, the dilaton potential should vanish $\left(\Lambda_{b}=0\right)$ in order to obtain the $4 \mathrm{D}$ Minkowski space, while $k_{1}$ is not determined by the equation of motion. ${ }^{4}$ On the other hand, $\hat{c}^{2}=0\left(k_{2}=0\right)$ corresponds to the familiar Randall-Sundrum (RS) model with the $\mathrm{AdS}_{5}$ bulk space. Also, $\hat{c}^{2}=1$ is a special point where $k_{1}=k_{2}$, which corresponds to the linear dilaton model (LD). Finally, the flat large extra dimension (LED) can be realized when either the dilaton potential vanishes $\left(\Lambda_{b}=0\right)$ or $\hat{c}^{2} \rightarrow \infty$ so that $k_{1}=0$. These critical points are summarized in figure 1.

The point $\hat{c}^{2}=1$ is important in another aspect. In fact, GLD with a finite $\hat{c}^{2}>1$ predicts a new KK structure which is distinctive from the KK spectra and couplings found in the RS, LD and LED scenarios. ${ }^{5}$ A schematic picture for $\hat{c}^{2}>1$ is given in figure 2. For convention, we will put our brane at $y=0$ and consider the positive $k_{1}$ solution in (2.11), meaning a positive $k$ of (2.8), because this turns out to be able to address the weak scale hierarchy problem. The crucial point is that the parameter $p$ of $(2.8)$ is then negative for $\hat{c}^{2}>1$. This means that the clockwork gear mass function $m(y)=e^{p y}$ shows exponentially decreasing profile over the extra dimension. Then the zero mode and lightest KK modes are localized near the distant brane $y=\pi R$ so as to have tiny couplings to us. This provides a hierarchy between the $4 \mathrm{D}$ Planck mass $M_{\mathrm{P}}$ and the $5 \mathrm{D}$ fundamental scale $M_{5}$ by $M_{5} \sim M_{\mathrm{P}} e^{-k \pi R}$ with the parameter $k$ of (2.8). The lightest KK modes' couplings are similarly suppressed. Since the lightest KK modes have much smaller mass scale $\left(M_{\mathrm{KK}}^{(1)} \sim M_{5} e^{-|p| \pi R}\right)$ compared to the 5D cutoff $M_{5}$, the model predicts the KK structure

\footnotetext{
${ }^{4}$ As we will discuss a specific example in section $6, k_{1}$ can be determined by a BPS condition for $\hat{c}^{2}=4$.

${ }^{5}$ Recently, novel collider phenomenology of the LD $\left(\hat{c}^{2}=1\right)$ scenario compared to RS and LED was extensively studied in [58]. The GLD models with $\hat{c}^{2}>1$ will be even distinctive from the LD scenario.
} 


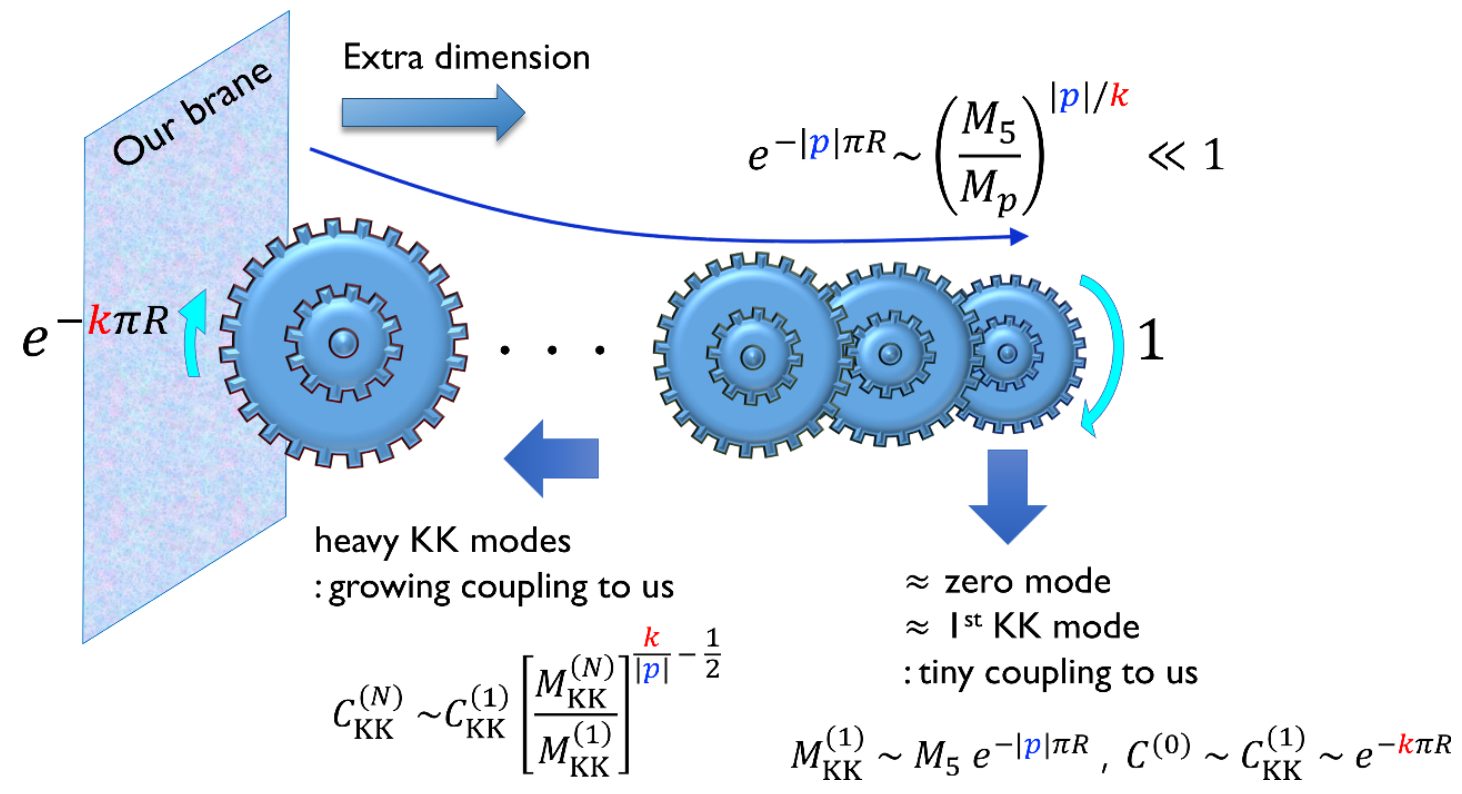

Figure 2. The KK structure for $\hat{c}^{2}>1$.

qualitatively similar to LED concerning the lightest modes. On the other hand, as for heavy $K K$ modes with masses near the $5 \mathrm{D}$ cutoff $M_{5}$, their couplings are substantially different from LED. In LED, all KK mode couplings are universally suppressed regardless of their masses. In GLD with a finite $\hat{c}^{2}>1$, however, heavier KK modes have larger couplings to us proportional to a certain power of their masses. This is because the heavy clockwork gears are closer to us as depicted in figure 2. This may entail interesting phenomenological consequences. We will give a more detailed discussion for the KK structure of $\hat{c}^{2}>1$ in section 4 .

In this paper, we are looking for a motivated UV completion of the GLD clockwork. The point $\hat{c}^{2}=1(\mathrm{LD})$ is known to be a $5 \mathrm{D}$ approximating theory of the gravity dual of the type II Little String Theory [59]. We now want to see whether there are more ways to generate clockworks from different types of string theory. As we will see in the next section, Hořava-Witten theory [5] (or heterotic M-theory) provides a direct realization of the GLD clockworks, especially for the region $\hat{c}^{2}>1$.

\section{Hořava-Witten model (minimal heterotic M-theory)}

The strongly coupled $E_{8} \times E_{8}$ heterotic M-theory may be effectively described in terms of 11-D supergravity with the bosonic part of the action given by

$$
\begin{aligned}
\mathcal{S}_{11}= & -\frac{1}{2 \kappa^{2}} \int_{\mathcal{M}^{11}} \mathrm{~d}^{11} x \sqrt{-g}\left(-\mathcal{R}+\frac{1}{24} G_{I J K L} G^{I J K L}+\frac{\sqrt{2}}{1728} \epsilon^{I_{1} I_{2} \ldots I_{11}} C_{I_{1} I_{2} I_{3}} G_{I_{4} \ldots I_{7}} G_{I_{8} \ldots I_{11}}\right) \\
& -\frac{1}{8 \pi \kappa^{2}}\left(\frac{\kappa}{4 \pi}\right)^{2 / 3} \sum_{i=1}^{2} \int_{\mathcal{M}_{(i)}^{10}} \mathrm{~d}^{10} x \sqrt{-g}\left(\operatorname{tr} F_{(i)}^{2}-\frac{1}{2} \operatorname{tr} \mathcal{R}^{2}\right) .
\end{aligned}
$$


In order to obtain $\mathcal{N}=1$ supersymmetry in $4 \mathrm{D}$ we compactify this theory on a CalabiYau (CY) complex 3-manifold $X^{6}$. We work in the "upstairs" approach in which $\mathcal{M}^{11}$ is a warped product manifold $\mathcal{M}^{4} \times X^{6} \times S^{1}$ where $\mathcal{M}^{4}$ is the $4 \mathrm{D}$ Minkowski space-time and the circle $S^{1}$ is parameterized by the 11-th coordinate $x^{11}$. If one chooses $x^{11}$ to be in the range $\left(-\pi r_{11}, \pi r_{11}\right]$ then all the fields must be symmetric or anti-symmetric and the Lagrangian must be invariant under parity transformation $x^{11} \rightarrow-x^{11}$. In the rest of the paper we will use the following conventions for the space-time indices: ${ }^{6} I, J, \ldots=$ $0, \ldots, 9,11 ; A, B, \ldots=4, \ldots, 9$ are tangent to $X^{6} ; \mu, \nu, \ldots=0,1,2,3$ are tangent to $\mathcal{M}^{4}$; $\alpha, \beta, \ldots=0,1,2,3,11$.

Two $E_{8}$ gauge groups with the field strengths $F_{(i)}$ are localized at two $10 \mathrm{D}$ branes $\mathcal{M}_{(i)}^{10}$ at $x_{(1)}^{11}=0$ and $x_{(2)}^{11}=\pi r_{11}$, respectively. By convention we choose the brane at $x^{11}=0$ to support the gauge sector containing that of the Standard Model. In order for this theory to be supersymmetric and anomaly-free the Bianchi identity for the field strength $G$ is modified such that

$$
\mathrm{d} G=-\frac{1}{2 \sqrt{2} \pi}\left(\frac{\kappa}{4 \pi}\right)^{2 / 3} \sum_{i}\left(\operatorname{tr} F_{(i)}^{2}-\frac{1}{2} \operatorname{tr} \mathcal{R}^{2}\right) \delta\left(x^{11}-x_{(i)}^{11}\right),
$$

in the leading order of the expansion in $\kappa^{2 / 3}$, which results in the following non-zero values of $G$ :

$$
G_{A B C D}=-\frac{\mu}{48} \epsilon_{A B C D}{ }^{E F} \omega_{E F}
$$

where $\omega_{E F}$ is the Kähler form on $X^{6}$ while $\mu$ is given by

$$
\mu \equiv \frac{\sqrt{2}}{\pi V_{0}}\left(\frac{\kappa}{4 \pi}\right)^{2 / 3} \int_{X^{6}} \omega \wedge\left(\operatorname{tr} F_{(1)} \wedge F_{(1)}-\frac{1}{2} \operatorname{tr} \mathcal{R} \wedge \mathcal{R}\right)
$$

and $V_{0} \equiv \int_{X} \mathrm{~d}^{6} x \sqrt{\operatorname{det} g_{A B}}$ is the zeroth order CY volume. Notice that $\mu$ is negative for the standard embedding of the spin connection in the gauge group (i.e. when $\operatorname{tr} F_{(1)}^{2}=\operatorname{tr} \mathcal{R}^{2}$ locally at the brane at $x^{11}=0$ ) but may be positive for some non-standard ones. A discussion of the possibilities can be found in [60].

The metric on $\mathcal{M}^{11}$ has the form

$$
\mathrm{d} s_{11}^{2}=(1+\hat{b}) \eta_{\mu \nu} \mathrm{d} x^{\mu} \mathrm{d} x^{\nu}+\left(g_{A B}+\hat{h}_{A B}\right) \mathrm{d} x^{A} \mathrm{~d} x^{B}+(1+\hat{\gamma})\left(\mathrm{d} x^{11}\right)^{2},
$$

where the corrections $\hat{b}, \hat{h}_{A B}$ and $\hat{\gamma}$ are functions of only $x^{11}$. The CY part simplifies to $\hat{h}_{A B}=\hat{h} g_{A B}$ if only the universal modulus is taken into account. Then, in the leading nontrivial order of the $\kappa^{2 / 3}$ expansion, the above corrections were found to be ${ }^{7}$ [61-65]

$$
\hat{b}=\hat{b}_{0} \mu\left|x^{11}\right|, \quad \hat{h}=\hat{h}_{0} \mu\left|x^{11}\right|, \quad \hat{\gamma}=\hat{\gamma}_{0} \mu\left|x^{11}\right|,
$$

with

$$
\hat{b}_{0}=-\hat{h}_{0}=-\frac{\hat{\gamma}_{0}}{2}=-\frac{\sqrt{2}}{24} .
$$

\footnotetext{
${ }^{6}$ Observe the difference with respect to section 2: the fifth coordinate of the 5D (sub)space was denoted by $x^{5}$ or $y$ and now is denoted by $x^{11}$.

${ }^{7}$ Of course $\hat{\gamma}$ may be changed by a reparametrization of $x^{11}$.
} 
The effective $4 \mathrm{D}$ Planck mass is given by

$$
\begin{aligned}
M_{\mathrm{P}}^{2} & =2 M_{11}^{9} \int_{0}^{\pi r_{11}} \mathrm{~d} x^{11} \int_{X^{6}} \mathrm{~d}^{6} x(1+\hat{b}) \sqrt{\operatorname{det}\left(g_{A B}+\hat{h}_{A B}\right)} \sqrt{1+\hat{\gamma}} \\
& \simeq 2 M_{11}^{9} V_{0} \int_{0}^{\pi r_{11}} \mathrm{~d} x^{11}\left(1+\frac{1}{2} \hat{\gamma}+\hat{b}+3 \hat{h}\right) \simeq 2 M_{11}^{9} V_{0} \pi R_{11}\left(1+\frac{1}{2} \mu \pi R_{11}\left|\hat{b}_{0}+3 \hat{h}_{0}\right|\right)
\end{aligned}
$$

where $M_{11} \equiv \kappa^{-2 / 9}$ is the $11 \mathrm{D}$ Planck scale and the integration is performed over the CY space $X^{6}$ and the 11 -th interval $I_{11} \equiv S^{1} / \mathbb{Z}_{2}$, and $\pi R_{11}$ denotes the physical length of $I_{11}$,

$$
\pi R_{11}=\int_{0}^{\pi r_{11}} \mathrm{~d} x^{11} \sqrt{1+\hat{\gamma}} \simeq \pi r_{11}\left(1+\frac{1}{4} \hat{\gamma}_{0} \mu \pi r_{11}\right)
$$

On the other hand, the 6D CY volume scales with $x^{11}$ as

$$
V\left(x^{11}\right)=\int_{X^{6}} \mathrm{~d}^{6} x \sqrt{\operatorname{det}\left(g_{A B}+h_{A B}\right)}=V_{0}(1+\hat{h})^{3} \simeq V_{0}\left(1+3 \mu \hat{h}_{0}\left|x^{11}\right|\right) .
$$

For negative $\mu$ (e.g. for the standard embedding) $V$ decreases with $\left|x^{11}\right|$ which results in an upper bound on the length of the 11-th dimension, $\pi R_{11}$. On the other hand, $V$ increases with $\left|x^{11}\right|$ for positive $\mu$. In such a case $R_{11}$ may be quite large and the hierarchy problem may be addressed. In the large $R_{11}$ limit the effective $4 \mathrm{D}$ Planck mass (3.8) reads

$$
M_{\mathrm{P}}^{2} \rightarrow \frac{\sqrt{2}}{12} \mu M_{11}^{9} V_{0}\left(\pi R_{11}\right)^{2}=\left[\frac{\sqrt{2}}{12}\left(\frac{\mu}{M_{11}}\right)\left(M_{11}^{6} V_{0}\right)\right] M_{11}^{4}\left(\pi R_{11}\right)^{2} .
$$

$V_{0}$ is equal to the $\mathrm{CY}$ volume at the observable brane at $x^{11}=0$ so it is related to the value of the GUT gauge coupling, namely $[61,63] V_{0} M_{11}^{6}=(4 \pi)^{2 / 3} \alpha_{\mathrm{GUT}}^{-1} \approx 135$. Assuming $\frac{1}{16 \pi^{2}} \int_{X^{6}} \omega \wedge\left(\operatorname{tr} F^{2}-\frac{1}{2} \operatorname{tr} \mathcal{R}^{2}\right) \simeq a V_{0}^{1 / 3}$, where $a$ is an order 1 constant, one may estimate the value of the ratio $\mu / M_{11} \simeq 0.5 a$. Using these relations among the massive parameters in the square bracket in (3.11) one gets

$$
M_{\mathrm{P}}^{2} \approx 8 a M_{11}^{2}\left(M_{11} \pi R_{11}\right)^{2}
$$

Large 11-th dimension may be used to address the hierarchy problem. For example for $\pi R_{11} \lesssim 100 \mu \mathrm{m}$ the fundamental mass scale $M_{11}$ of order $1.3 a^{-1 / 4} \mathrm{TeV}$ is enough to obtain the correct value of $M_{\mathrm{P}}$.

Notice that the effective $4 \mathrm{D}$ Planck mass scales as $M_{\mathrm{P}}^{2} \sim M_{11}^{4}\left(\pi R_{11}\right)^{2}$. Such relation is typical for models with two flat extra dimensions. In the present case it comes from seven extra dimensions: one large (and flat), $x^{11}$, and six curved, $x^{A}$, with the 6 -volume changing linearly with $x^{11}$.

We consider situation when the 11-th dimension is much larger than the remaining six compact dimensions. So, an effective $5 \mathrm{D}$ description is adequate in such a limit. In the reduction to $5 \mathrm{D}$ we will retain only the $\mathrm{CY}$ zero modes and universal moduli while keeping the non-zero mode (3.3). The 11D metric may be written in the form

$$
\mathrm{d} s_{11}^{2}=\hat{V}^{-2 / 3} g_{\alpha \beta} \mathrm{d} x^{\alpha} \mathrm{d} x^{\beta}+\hat{V}^{1 / 3} g_{A B} \mathrm{~d} x^{A} \mathrm{~d} x^{B} .
$$


The dependence of the CY volume on $5 \mathrm{D}$ coordinates $x^{\alpha}$ is only through the volume modulus field $\hat{V} \equiv V / V_{0}$. The factor $\hat{V}^{-2 / 3}$ in front of $g_{\alpha \beta} \mathrm{d} x^{\alpha} \mathrm{d} x^{\beta}$ is introduced to obtain the 5D Einstein frame after reduction. In this section we consider the simplest version of the heterotic M-theory so in the reduction we use only the following components of the 3-form field $C_{I J K}$ :

$$
C_{\alpha \beta \gamma}, \quad C_{\alpha A B}=\frac{1}{6} \mathcal{A}_{\alpha} \omega_{A B}, \quad C_{A B C}=\frac{1}{6} \xi \omega_{A B C},
$$

where $\omega_{A B C}$ is the harmonic $(3,0)$ form on the CY space. The 3 -form field $C_{\alpha \beta \gamma}$ can be dualized to a scalar $\sigma$. The resulting theory is the minimal ${ }^{8}$ supergravity in $5 \mathrm{D}$ with the universal hypermultiplet $\left(\hat{V}, \sigma, \xi, \bar{\xi}, \zeta^{i}\right)$ and the gravity multiplet $\left(g_{\alpha \beta}, \mathcal{A}_{\alpha}, \psi_{\alpha}^{i}\right)$ where $\zeta^{i}$ and $\psi_{\alpha}^{i}$ are the hypermultiplet fermions and gravitini, respectively, and $i=1,2$.

Integrating the $11 \mathrm{D}$ action (3.1) over the CY space $X^{6}$ one can obtain the effective 5D action. Such reduction retaining the above mentioned fields and the gauge fields localized at the branes was performed in [65]. The relevant part for us of this effective action, describing the gravity-modulus system reads

$$
\begin{aligned}
\mathcal{S}_{5}= & -\frac{1}{2 \kappa_{5}^{2}} \int_{\mathcal{M}^{5}} \mathrm{~d}^{5} x \sqrt{-g}\left[-\mathcal{R}_{5}+\frac{1}{2} \hat{V}^{-2} \partial_{\alpha} \hat{V} \partial^{\alpha} \hat{V}+\frac{\mu^{2}}{192} \hat{V}^{-2}\right] \\
& -\frac{1}{2 \kappa_{5}^{2}}\left\{\frac{\sqrt{2}}{4} \int_{\mathcal{M}_{(1)}^{4}} \mathrm{~d}^{4} x \sqrt{-g} \mu \hat{V}^{-1}-\frac{\sqrt{2}}{4} \int_{\mathcal{M}_{(2)}^{4}} \mathrm{~d}^{4} x \sqrt{-g} \mu \hat{V}^{-1}\right\},
\end{aligned}
$$

where $\kappa_{5}^{2}=\kappa^{2} / V_{0}$. Notice that the non-zero mode of $G(3.3)$ determines the interaction terms between the graviton and CY volume modulus. Therefore, this mode is responsible for characteristic low energy predictions of heterotic M-theory distinctive from other higher dimensional supergravity/gravity theories, found e.g in [66, 67].

We would like to analyze the above action in the context of GLD models discussed in [4] and summarized in the previous section. Replacing the volume modulus $\hat{V}$ with scalar $S$ defined by $\hat{V} \equiv \exp (\sqrt{2} S)$ we may rewrite the above $5 \mathrm{D}$ action in the general form $(2.9)$

$$
\begin{aligned}
\mathcal{S}_{5}= & \frac{1}{\kappa_{5}^{2}} \int_{\mathcal{M}^{5}} \mathrm{~d}^{5} x \sqrt{-g}\left[\frac{1}{2} \mathcal{R}_{5}-\frac{1}{2} \partial_{\alpha} S \partial^{\alpha} S-\Lambda_{b} e^{-(2 \hat{c} / \sqrt{3}) S}\right] \\
& -\frac{1}{\kappa_{5}^{2}} \sum_{i} \int_{\mathcal{M}_{(i)}^{4}} \mathrm{~d}^{4} x \sqrt{-g} \Lambda_{(i)} e^{-(\hat{c} / \sqrt{3}) S}
\end{aligned}
$$

with

$$
\hat{c}^{2}=6, \quad \Lambda_{b}=\frac{\mu^{2}}{384}, \quad \Lambda_{(1)}=-\Lambda_{(2)}=\frac{\mu}{4 \sqrt{2}} .
$$

Thus the dimensional reduction of Horava-Witten model directly realizes a GLD model with $\hat{c}^{2}=6$, and the bulk potential is positive as required for $\hat{c}^{2}>4$ as discussed in section 2. Also remarkably, the boundary dilaton potentials satisfy the relation (2.10) to obtain the 4D Minkowski solution. This is due to the modified Bianchi identity (3.2) for

\footnotetext{
${ }^{8} \mathcal{N}=1$ SUSY from the 5D point of view which may lead to $\mathcal{N}=2$ in 4 D after compactification on $S^{1}$.
} 
the 11D theory to be locally supersymmetric and anomaly free, which implies that the boundary potentials induced by non-zero magnetic flux $\mu$ must have equal magnitude and opposite sign.

We can reproduce the leading order result (3.8) of the $11 \mathrm{D}$ action by the effective $5 \mathrm{D}$ GLD action (3.16) as well. From the 5D background geometry (2.7) with the parameters (2.11) and (2.8), it comes out to be

$$
M_{\mathrm{P}}^{2}=\frac{1}{\kappa_{5}^{2} k}\left(\exp \left(2 k \pi r_{11}\right)-1\right)=M_{11}^{9} V_{0}\left(\frac{\sqrt{2}}{12} \mu\left(\pi R_{11}\right)^{2}+2\left(\pi R_{11}\right)\right),
$$

where $\pi r_{11}$ is the 11-th coordinate position of the second brane in the coordinate frame used in (2.7), and it is used that

$$
\pi R_{11}=\int_{0}^{\pi r_{11}} \mathrm{~d} x^{11} \hat{V}^{-1 / 3} e^{k_{2} x^{11}}=\frac{3}{2 k_{2}}\left(\exp \left(\frac{2}{3} k_{2} \pi r_{11}\right)-1\right)
$$

from (3.13) in order to relate the coordinate radius $r_{11}$ to the physical radius $R_{11}$. Thus, the $11 \mathrm{D}$ leading order result can be obtained as the exact result of the $5 \mathrm{D}$ effective theory as pointed out in [65].

\section{Masses and couplings of Kaluza-Klein (KK) states}

As emphasized in section $2, \hat{c}^{2}=6>1$ will exhibit a new KK structure. The results of the GLD KK spectrum and couplings analysis presented in [4] may be applied to the lightest KK excitations. In terms of the parameters defined in section 2, the $n$-th KK mode mass $M_{n}$ and coupling $C_{n}$ to our brane turn out to be approximated as

$$
\begin{aligned}
M_{n} & \approx\left(n-\frac{1}{4}+\frac{k}{2|p|}\right) \pi|p| \exp \left(-|p| \pi r_{11}\right), \\
C_{n} & \approx \frac{\sqrt{\pi|p| / k}}{\Gamma(k /|p|)}\left[\frac{\pi}{2}\left(n-\frac{1}{4}+\frac{k}{2|p|}\right)\right]^{\frac{k}{|p|}-\frac{1}{2}} \exp \left(-k \pi r_{11}\right),
\end{aligned}
$$

for $p \lesssim-k$ with a positive $k$, where $n=1,2, \ldots$. We have confirmed that these formulae are valid even for heavy KK modes up to $n \sim \exp \left(|p| \pi r_{11}\right) .{ }^{9}$ In minimal heterotic M-theory, the parameters $k$ and $p$ are equal to

$$
\begin{aligned}
& k= \pm\left(1+\frac{\hat{c}^{2}}{2}\right) \sqrt{\frac{2}{3}\left(\frac{\Lambda_{b}}{\hat{c}^{2}-4}\right)}=\frac{\sqrt{2}}{12} \mu, \\
& p=\operatorname{sgn}(k)\left(1-\hat{c}^{2}\right) \sqrt{\frac{2}{3}\left(\frac{\Lambda_{b}}{\hat{c}^{2}-4}\right)}=-\frac{5 \sqrt{2}}{48} \mu,
\end{aligned}
$$

since $\hat{c}^{2}=6$ and $\Lambda_{b}=\mu^{2} / 384$ from (3.17), and the sign \pm is determined by the sign of $\mu$. Thus for a positive $\mu$, which is necessary to address the hierarchy problem, the condition

\footnotetext{
${ }^{9}$ The coupling $C_{n}$ for an exponentially large $n\left(n \lesssim \exp \left(|p| \pi r_{11}\right)\right)$ can be different from the formula (4.2) by a factor of order one. But we will not be precise on this factor, since it is not important for our discussion.
} 
$p \lesssim-k$ is satisfied to apply the above approximate formulae for the KK spectrum and couplings. As was argued in section 2, the lightest KK masses are exponentially suppressed compared to the 5D fundamental scale $\kappa_{5}^{-2 / 3} \sim M_{11} \sim \mu$ by the factor $\exp \left(-|p| \pi r_{11}\right)$ with the negative $p$ in (4.4) for $\hat{c}^{2}>1$.

Using eq. (3.19), one can express the above KK spectrum in terms of the length of the 11-th dimension, $\pi R_{11}$. The result (valid for positive $\mu$ ) reads

$$
M_{n} \approx\left(n+\frac{3}{20}\right) \frac{5 \sqrt{2} \pi}{48} \mu\left(1+\frac{\sqrt{2}}{12} \mu \pi R_{11}\right)^{-5 / 4} .
$$

Using the relation (3.18) between the Planck mass and $R_{11}$, we may rewrite (4.5) in the large $R_{11}$ limit

$$
\begin{aligned}
M_{n} & \approx\left(n+\frac{3}{20}\right) \frac{5 \sqrt{2} \pi}{48}(6 \sqrt{2})^{5 / 8}\left(M_{11}^{6} V_{0}\right)^{5 / 8}\left(\frac{\mu}{M_{11}}\right)^{3 / 8} M_{11}\left(\frac{M_{11}}{M_{\mathrm{P}}}\right)^{5 / 4} \\
& \approx 29 n a^{3 / 8} M_{11}\left(\frac{M_{11}}{M_{\mathrm{P}}}\right)^{5 / 4} .
\end{aligned}
$$

In the case of $N$ large flat extra dimensions (LED), the KK masses scale as

$$
M_{\mathrm{LED},\left\{n_{i}\right\}}^{2}=\left(\sum_{i} n_{i}^{2}\right) M_{*}^{2}\left(\frac{M_{*}}{M_{\mathrm{P}}}\right)^{2 / N}
$$

where $\left\{n_{i}\right\}$ is the set of $N$ numbers describing excitations along each dimension and $M_{*}$ is the fundamental mass scale of the model. Comparing the above formula with the spectrum (4.6) for large $n$, we see that asymptotically the KK spectrum of the heterotic Mtheory is in this case similar ${ }^{10}$ to that of $N=8 / 5=1.6$ large extra dimensions.

The GLD clockwork from the simplest version of the heterotic M-theory thus resembles fractional large extra dimensions in terms of the KK spectrum, while the hierarchy problem is solved as in the case of two large flat extra dimensions. Moreover, the KK coupling is predicted to be quite different from LED. To see this, let us express (4.2) in terms of the KK mass (4.1):

$$
C_{n} \approx \frac{\sqrt{\pi|p| / k}}{\Gamma(k /|p|)}\left(\frac{M_{n}}{2|p|}\right)^{\frac{k}{|p|}-\frac{1}{2}} e^{-\frac{1}{2}|p| \pi r_{11}} \approx 97 a^{-1 / 2}\left(\frac{M_{n}}{M_{1}}\right)^{3 / 10}\left(\frac{M_{11}}{M_{\mathrm{P}}}\right),
$$

where $M_{1}$ is the first KK mass. So the coupling (4.8) is similar to the LED KK coupling $C_{\mathrm{LED},\left\{n_{i}\right\}}=M_{*} / M_{\mathrm{P}}$ for the light KK states with $M_{n} \sim M_{1}$. But it grows for heavier KK states. For instance, if we consider a KK state whose mass is near the fundamental cut-off $M_{n} \sim M_{11}$, the coupling becomes

$$
C_{n}\left(M_{n} \simeq M_{11}\right) \approx 34 a^{-49 / 80}\left(\frac{M_{11}}{M_{\mathrm{P}}}\right)^{5 / 8} \gg \frac{M_{11}}{M_{\mathrm{P}}} .
$$

\footnotetext{
${ }^{10}$ Of course, similar are mass levels but not their multiplicities — all levels in the considered M-theory model are non-degenerate.
} 
The phenomenological implications of this novel KK-structure have to be studied further. If the scale $M_{11}$ is small enough this might lead to the production of KK-excitations of the graviton at high energy colliders like the Large Hadron Collider (LHC) at CERN. To estimate this production, we consider the branching ratio for emitting a KK-graviton of mass scale $M_{n}$ in a physical process with available energy $E$. This can be estimated as

$$
\operatorname{Br}\left(M_{n}\right) \sim E^{2}\left(\frac{C_{n}}{M_{11}}\right)^{2}\left(\frac{M_{n}}{\Delta M_{n}}\right) \sim\left(\frac{E}{M_{11}}\right)^{2}\left(\frac{M_{n}}{M_{11}}\right)^{8 / 5},
$$

where $\Delta M_{n}$ is the mass gap of the KK-states and the factor $\left(M_{n} / \Delta M_{n}\right)$ accounts for the multiplicity of the KK states. Thus for a collison energy $E$ comparable to the string scale $M_{11}$, KK-gravitons of mass $\sim M_{11}$ may be produced at colliders and the spectrum can be analysed. As we pointed out above, the string scale $M_{11}$ can be as low as a few TeV for the models under consideration.

\section{$5 \quad$ Heterotic M-theory with vector multiplets}

In the previous two sections, we have shown that the $5 \mathrm{D}$ effective theory of heterotic Mtheory in its simplest form realizes the GLD with $\hat{c}^{2}=6$. The model was obtained based on the CY volume modulus and graviton interactions. Now we want to consider a role of more Kähler moduli besides the $\mathrm{CY}$ volume modulus i.e. we consider models compactified on CY space with the Hodge number $h_{(1,1)}>1$. We will show that in the simplest cases, two more solutions $\hat{c}^{2}=7,10$ are obtained.

For a Calabi-Yau three-fold $X^{6}$ with the Hodge number $h_{(1,1)}$, the internal metric $g_{a \bar{b}}$ and Kähler form $\omega_{a \bar{b}}=i g_{a \bar{b}}(a, b, \ldots$ and $\bar{a}, \bar{b}, \ldots$ are holomorphic and anti-holomorphic indices on the CY space, respectively) can be expanded with a basis $\omega_{i} \in H^{(1,1)}\left(X^{6}\right)$ $\left(i=1, \ldots, h_{(1,1)}\right)$,

$$
\omega=t^{i} \omega_{i}
$$

where $t^{i}$ are the Kähler moduli. Then the internal CY space volume $V$ is

$$
V=\int_{X^{6}} \mathrm{~d}^{6} x \sqrt{\operatorname{det} g_{A B}}=\frac{1}{3 !} \int_{X^{6}} \omega \wedge \omega \wedge \omega=\frac{1}{6} V_{0} d_{i j k} t^{i} t^{j} t^{k},
$$

with the CY intersection numbers

$$
d_{i j k} \equiv \frac{1}{V_{0}} \int_{X^{6}} \omega_{i} \wedge \omega_{j} \wedge \omega_{k}
$$

If we rescale $t^{i}=\hat{V}^{1 / 3} X^{i}$, we can separate the volume modulus $\hat{V}\left(\equiv V / V_{0}\right)$ from the other Kähler moduli $X^{i}$ which satisfy the constraint

$$
1=\frac{1}{6} d_{i j k} X^{i} X^{j} X^{k}
$$

so that there are $h_{(1,1)}-1$ independent Kähler moduli apart from the volume modulus. 
The 3-form field component $C_{\alpha A B}$ in (3.14) can be also expanded as

$$
C_{\alpha A B}=\frac{1}{6} \mathcal{A}_{\alpha}^{i} \omega_{i A B}
$$

Then in the dimensional reduction to the $5 \mathrm{D}$ effective theory, one of the $5 \mathrm{D}$ vectors $\mathcal{A}_{\alpha}^{i}(i=$ $\left.1, \ldots, h_{(1,1)}\right)$ is identified as the graviphoton in the $5 \mathrm{D}$ gravity multiplet, and the remaining $\left(h_{(1,1)}-1\right) 5 \mathrm{D}$ vectors compose $\left(h_{(1,1)}-1\right) 5 \mathrm{D}$ vector multiplets with the Kähler moduli $X^{i}$. Thus in the resulting 5D theory, we have vector multiplets in addition to the universal hypermultiplet and the gravity multiplet considered in the previous section.

In fact, we can also have $h_{(2,1)} 5 \mathrm{D}$ hypermultiplets constructed out of $h_{(2,1)}$ complex structure moduli $g_{a b}$ and 3 -form field components $C_{a b \bar{c}}$. However, it turns out that these hypermultiplets do not contribute to the scalar potential, so they are irrelevant for our discussion.

The reduction of the $11 \mathrm{D}$ action (3.1) to the $5 \mathrm{D}$ effective action retaining the additional vector multiplets was elaborated in [68]. The part relevant for us describes the following gravity-Kähler moduli system:

$$
\begin{aligned}
\mathcal{S}_{5}= & -\frac{1}{2 \kappa_{5}^{2}} \int_{\mathcal{M}^{5}} \sqrt{-g}\left[-\mathcal{R}_{5}+\frac{1}{2} \hat{V}^{-2} \partial_{\alpha} \hat{V} \partial^{\alpha} \hat{V}+G_{i j}(X) \partial_{\alpha} X^{i} \partial^{\alpha} X^{j}+\frac{1}{128} \hat{V}^{-2} G^{i j}(X) \mu_{i} \mu_{j}\right] \\
& -\frac{1}{2 \kappa_{5}^{2}}\left\{\frac{\sqrt{2}}{4} \int_{\mathcal{M}_{(1)}^{4}} \sqrt{-g} \hat{V}^{-1} \mu_{i} X^{i}-\frac{\sqrt{2}}{4} \int_{\mathcal{M}_{(2)}^{4}} \sqrt{-g} \hat{V}^{-1} \mu_{i} X^{i}\right\}
\end{aligned}
$$

where $X^{i}\left(i=1, \ldots, h_{(1,1)}\right)$ are subject to the constraint (5.4), and

$$
G_{i j}(X)=-\frac{\hat{V}^{2 / 3}}{2} \frac{\partial^{2}}{\partial t^{i} \partial t^{j}} \ln \hat{V}=-\frac{1}{2}\left[d_{i j k} X^{k}-\frac{1}{4}\left(d_{i l m} X^{l} X^{m}\right)\left(d_{j n p} X^{n} X^{p}\right)\right]
$$

and the previous flux parameter $\mu$ is generalized to

$$
\mu_{i} \equiv \frac{\sqrt{2}}{\pi V_{0}}\left(\frac{\kappa}{4 \pi}\right)^{2 / 3} \int_{X} \omega_{i} \wedge\left(\operatorname{tr} F_{(1)} \wedge F_{(1)}-\frac{1}{2} \operatorname{tr} \mathcal{R} \wedge \mathcal{R}\right)
$$

The important part of the above action is the bulk and boundary scalar potentials arising from non-zero flux parameters $\mu_{i}$. In particular, the bulk potential derives from non-zero values of the internal components of $G$ as a solution to the modified Bianchi identity (3.2):

$$
G_{A B C D}=-\frac{\hat{V}^{2 / 3}}{32} G^{i j} \mu_{i} \epsilon_{A B C D}{ }^{E F} \omega_{j E F} \epsilon\left(x^{11}\right) .
$$

As a simple choice, let us first consider $h_{(1,1)}=2$ and the CY intersection number $d_{112} \neq 0$ while the other components $d_{i j k}$ vanish. Then the constraint (5.4) is

$$
d_{112}\left(X^{1}\right)^{2} X^{2}=2 .
$$

So we can write

$$
X^{1}=\frac{1}{\beta} e^{-b S_{1}}, \quad X^{2}=\frac{1}{\beta} e^{2 b S_{1}},
$$


where $\beta=\left(d_{112} / 2\right)^{1 / 3}$, and it turns out that $b=1 / \sqrt{3}$ to canonically normalize $S_{1}$. On the other hand, we replace the volume modulus $\hat{V}$ with scalar $S_{V}$ defined by $\hat{V}=\exp \left(\sqrt{2} S_{V}\right)$ as before, which corresponds to a canonical normalization for $S_{V}$. Then the scalar potential turns out to be

$$
\begin{aligned}
V_{\text {bulk }} & =\frac{1}{256 \kappa_{5}^{2}} e^{-2 \sqrt{2} S_{V}}\left[\frac{\mu_{1}^{2}}{\beta^{2}} e^{-\frac{2}{\sqrt{3}} S_{1}}+2 \frac{\mu_{2}^{2}}{\beta^{2}} e^{\frac{4}{\sqrt{3}} S_{1}}\right], \\
V_{\text {boundary }} & =\frac{\sqrt{2}}{8 \kappa_{5}^{2}} e^{-\sqrt{2} S_{V}}\left[\frac{\mu_{1}}{\beta} e^{-\frac{1}{\sqrt{3}} S_{1}}+\frac{\mu_{2}}{\beta} e^{\frac{2}{\sqrt{3}} S_{1}}\right]\left[\delta\left(x_{11}\right)-\delta\left(x_{11}-\pi r_{11}\right)\right] .
\end{aligned}
$$

If both $\mu_{1}$ and $\mu_{2}$ are non-zero, $S_{1}$ is stabilized at $\sqrt{3} S_{1}=\ln \left(\mu_{1} / 2 \mu_{2}\right)$. Then we recover the GLD potential (3.16) of the previous section with the GLD dilaton $S$ identified as $S_{V}$ and $\mu=3\left(\mu_{1}^{2} \mu_{2} / 2 d_{112}\right)^{1 / 3}$. So in this case, $\hat{c}^{2}=6$ as before. On the other hand, if one of the flux parameters $\mu_{i}$ vanishes, one can find a new solution. If $\mu_{1}=0$, a new run-away direction $-2 \sqrt{2} S_{V}+4 S_{1} / \sqrt{3}$ appears in the bulk potential, which is to be identified as the GLD dilaton $S$. In other words, we obtain the GLD action (2.9) with

$$
\begin{aligned}
\frac{\hat{c}}{\sqrt{3}} S & =\sqrt{2} S_{V}-\frac{2}{\sqrt{3}} S_{1}, \\
\hat{c}^{2} & =3\left[(\sqrt{2})^{2}+\left(\frac{2}{\sqrt{3}}\right)^{2}\right]=10,
\end{aligned}
$$

where $\hat{c}$ is determined to canonically normalize $S$ as in (5.15). Therefore, we find another GLD with $\hat{c}^{2}=10$ and $\Lambda_{b}=\left(2 / d_{112}\right)^{2 / 3} \mu_{2}^{2} / 128$, while the boundary potential satisfies the condition (2.10) for the $4 \mathrm{D}$ Minkowski background. Note that the bulk potential is positive as required for $\hat{c}^{2}>4$.

Another new solution is obtained when $\mu_{2}=0$. Similarly to $\mu_{1}=0$ case, we get the GLD action (2.9), this time with

$$
\begin{aligned}
\frac{\hat{c}}{\sqrt{3}} S & =\sqrt{2} S_{V}+\frac{1}{\sqrt{3}} S_{1}, \\
\hat{c}^{2} & =3\left[(\sqrt{2})^{2}+\left(\frac{1}{\sqrt{3}}\right)^{2}\right]=7 .
\end{aligned}
$$

This is a GLD with $\hat{c}^{2}=7$ and $\Lambda_{b}=\left(2 / d_{112}\right)^{2 / 3} \mu_{1}^{2} / 256$ while satisfying the boundary condition (2.10). The bulk potential is positive as well for $\hat{c}^{2}>4$.

The above solutions have different scaling for the hierarchy of the mass scales compared to the case without vector multiplets. Generalizing the formulae (3.18) and (3.19), it turns out that ${ }^{11}$

$$
M_{\mathrm{P}}^{2} \sim M_{11}^{2} \times\left(\pi R_{11} M_{11}\right)^{\left(\hat{c}^{2}+2\right) /\left(\hat{c}^{2}-2\right)} .
$$

Therefore, $\hat{c}^{2}=7,10$ correspond to $1.8,1.5$ flat extra dimensions, respectively. ${ }^{12}$ Analogously to the previous case, the corresponding KK spectra are matched to different

\footnotetext{
${ }^{11}$ This formula is strictly valid only for $\hat{c}^{2} \geq 6$ - see eq. (5.20) below.

${ }^{12}$ For smaller effective number of such extra dimensions larger values of $M_{11}$ are necessary to get the correct value of $4 \mathrm{D}$ Planck mass. For $\pi R_{11} \sim 100 \mu \mathrm{m}$ the $11 \mathrm{D}$ fundamental scale must be of order $\mathcal{O}(10) \mathrm{TeV}$ for $\hat{c}^{2}=7$ and $\mathcal{O}(100) \mathrm{TeV}$ for $\hat{c}^{2}=10$.
} 
(fractional) number of extra dimensions. For large $n$ the spectrum is similar to that of $N=3 / 2=1.5$ large flat extra dimensions for $\hat{c}^{2}=7$ and $N=4 / 3$ for $\hat{c}^{2}=10$. Generally the number of extra dimensions $N$ corresponding to KK spectrum is related to $\hat{c}$ by the following formula

$$
N=\frac{\hat{c}^{2}+2}{\hat{c}^{2}-1} .
$$

One should note that for heterotic M-theory for which $\hat{c}^{2} \geq 6$ (see eq. (5.20) and discussion below) both effective numbers of dimensions, one related to the hierarchy of scales in eqs. (5.18) and another related to the KK spectrum given by (5.19), are monotonically decreasing functions of $\hat{c}^{2}$.

We have therefore found two more GLD clockwork solutions $\hat{c}^{2}=7,10$ by the example of $h_{1,1}=2$ with $d_{112} \neq 0$ and $d_{i j k}=0$ otherwise. By a similar procedure, one can find that the same solutions are obtained for another simple example of $h_{1,1}=3$ and $d_{123} \neq 0$ while $d_{i j k}=0$ otherwise. Although we do not prove it rigorously, it seems quite generic that we recover the solution $\hat{c}^{2}=6$ when all flux parameters $\mu_{i}$ are non-zero, because the Kähler moduli involved in the vector multiplets are stabilized so that only the volume modulus plays the role of the GLD dilaton. On the other hand, if one of the flux parameters vanishes, a new run-away direction like (5.14) or (5.16) appears in the bulk potential so as to be identified as the GLD dilaton with a new $\hat{c}^{2}$. At any rate, the possibility to get more different solutions from higher $h_{1,1}$ or more complicated $d_{i j k}$ is still open.

From the above example, one can observe that there is a lower bound for $\hat{c}^{2}$ :

$$
\hat{c}^{2} \geq 3(\sqrt{2})^{2}=6
$$

This bound is due to the presence of the volume modulus $S_{V}$. The effect of the other Kähler moduli $S_{i}$ either does not change or increases $\hat{c}^{2}$. In fact, this is a generic lower bound for heterotic M-theory, since the general scalar potential in (5.6) is multiplied by the overall volume modulus factor. Therefore, $\operatorname{LD}\left(\hat{c}^{2}=1\right)$ or $\mathrm{RS}\left(\hat{c}^{2}=0\right)$ scenarios cannot be realized in heterotic M-theory. Does this mean that they cannot be consistently embedded in string theory? The next section is devoted to a discussion of this question.

\section{Can we avoid the heterotic M-theory bound?}

In the previous section, we derived the lower bound $\hat{c}^{2} \geq 6$ in the framework of heterotic Mtheory. It was observed that this bound is due to the presence of the CY volume modulus. With a compactification on a CY manifold we arrive at a 5D theory and we might try to understand the existence of such a bound in terms of 5D supergravity as well. Since the $\mathrm{CY}$ volume modulus is contained in the $5 \mathrm{D}$ universal hypermultiplet, this bound will be related to the presence of that multiplet. If we want to avoid the bound we have to discuss the role and coupling of the universal hypermultiplet in detail.

As pointed out in $[65,68,69]$, a non-zero $G$-flux in heterotic M-theory and/or 11D supergravity leads to gauging the resulting $5 \mathrm{D}$ effective supergravity along the axion $\sigma$ direction, which is the dual of the 3 -form component $C_{\alpha \beta \gamma}$. So the bulk scalar potential 
can be written in terms of a gauged 5D supergravity,

$$
V_{\text {bulk }}=-2 G^{i j}(X) \operatorname{tr} \mathcal{P}_{i} \mathcal{P}_{j}+4 X^{i} X^{j} \operatorname{tr} \mathcal{P}_{i} \mathcal{P}_{j}+\frac{1}{2} X^{i} X^{j} h_{u v} k_{i}^{u} k_{j}^{v}
$$

with the Killing vector $k_{i}=\left(\mu_{i} / 4\right) \partial_{\sigma}$ and the Killing prepotential

$$
\mathcal{P}_{i}=\left(\begin{array}{cc}
\frac{i}{32 \hat{V}} \mu_{i} & 0 \\
0 & -\frac{i}{32 \hat{V}} \mu_{i}
\end{array}\right)
$$

and $h_{u v}$ is the metric for the universal hypermultiplet $q^{u}=(\hat{V}, \sigma, \xi, \bar{\xi})$ in which $h_{\sigma \sigma}=$ $1 /\left(4 \hat{V}^{2}\right)$. The first two terms in the potential are contributions from the gravity and vector multiplets, while the last term is from the universal hypermultiplet. Now the last term cancels the second term so that the bulk potential is given by the first term alone. One can see that this first term is the same as the bulk potential in (5.6). Observe that this term is non-negative. As shown in figure 1 this implies that $\hat{c}^{2}$ has to be greater than 4 if a GLD clockwork solution exists (as explained in section 2).

In the framework of 5D supergravity, however, there exists previous work $[56,57]$ that describes clockwork systems with $\hat{c}^{2}<4$. The models considered there were constructed exclusively in the presence of vector multiplets. To make contact with these investigations we have to take our 5D system described above and remove the universal hypermultiplet. From the viewpoint of the underlying higher-dimensional string theory this might be problematic, as the universal hypermultiplet is an important ingredient in the compactifications of heterotic M-theory (and 11D M-theory as well). Let us nonetheless consider the case where the universal hypermultiplet is removed from the system. This corresponds to set the Killing vector $k_{i}=0$ and the Killing prepotential $\mathcal{P}_{i}=$ const. Since the third contribution in (6.1) vanishes, the second term now contributes to the bulk potential. Because this second term is non-positive, it can make the bulk potential negative so that $\hat{c}^{2}<4$ can be realized. Therefore, the heterotic lower bound for $\hat{c}^{2}$ can be avoided if the universal hypermultiplet is decoupled. However, we should stress that it is impossible to decouple the universal hypermultiplet in heterotic M-theory, because it is constrained by the boundary gauge couplings of $E_{8} \times E_{8}$, e.g. by perturbativity of the gauge couplings.

As an alternative we instead might consider M-theory (in contrast to heterotic Mtheory) in the limit of 11D supergravity and hope that such a situation can be realized there [56]. It is, however, not clear to us, how this can happen. The situation might just be a realization of the clockwork in 5D supergravity without a meaningful embedding in 11D M-theory.

Let us nonetheless discuss the phenomenological consequences in the absence of the universal hypermultiplet. As discussed above, this makes the second term in (6.1) contribute to the bulk potential in addition to the first term. If we again consider the example of the previous section $h_{(1,1)}=2$ and $d_{112} \neq 0$ while the other $d_{i j k}=0$, the bulk potential becomes

$$
V_{\text {bulk }}=\frac{1}{256 \kappa_{5}^{2}} \hat{V}^{-2}\left[-\frac{\mu_{1}^{2}}{\beta^{2}} e^{-\frac{2}{\sqrt{3}} S_{1}}-4 \frac{\mu_{1} \mu_{2}}{\beta^{2}} e^{\frac{1}{\sqrt{3}} S_{1}}\right]
$$


where $\hat{V}$ is a constant. Notice that the bulk potential is now negative. This is the same potential as found by $[56,57]$ in the context of the LD clockwork realization through $5 \mathrm{D}$ gauged supergravity with vector multiplets. As discussed there, if both $\mu_{1}$ and $\mu_{2}$ are non-zero, there is an extremum of the potential where $S_{1}$ is stabilized. This realizes the RS scenario $\left(\hat{c}^{2}=0\right)$ with negative bulk potential. On the other hand, if $\mu_{2}=0$ (and $\mu_{1} \neq 0$ ), $S_{1}$ becomes a run-away direction which realizes the GLD action (2.9) with

$$
S=S_{1} \text { and } \hat{c}^{2}=1
$$

corresponding to the linear dilaton case (LD). If $\mu_{1}=0$, the bulk potential vanishes. Still, a non-zero warping along the 11-th dimension can be obtained when $\mu_{2} \neq 0$ as discussed in [56]. Interestingly, the resultant background solution turns out to correspond to the GLD clockwork solution with

$$
S=S_{1} \text { and } \hat{c}^{2}=4
$$

while $\Lambda_{b}=0$ and $k_{1}$ is determined by $\mu_{2}$. This exhausts the solutions obtained in the case $h_{(1,1)}=3$ and $d_{123} \neq 0$ while the other $d_{i j k}=0$.

We have thus seen that in the absence of the universal hypermultiplet in the $5 \mathrm{D}$ supergravity theory, the heterotic bound $\hat{c}^{2} \geq 6$ can be avoided. In the presence of vector multiplets one can construct the solutions $\operatorname{RS}\left(\hat{c}^{2}=0\right)$ as well as $\operatorname{LD}\left(\hat{c}^{2}=1\right)$ and a third solution $\left(\hat{c}^{2}=4\right)$. The possibility to obtain other GLD clockwork solutions with higher $h_{(1,1)}$ or more complicated $d_{i j k}$ is the subject of further investigations.

\section{Conclusions and outlook}

GLD models provide a 2-parameter class of potential solutions to the weak- or axion-scale hierarchy problems. Depending on the parameters, the models differ in the properties of KK-masses and couplings. The GLD set-up has been discussed in a bottom-up construction and it remains to be seen whether there is a consistent UV-completion in the framework of string theory.

The results of our investigations are summarized in figure 3 , which indicate that such a valid UV-completion might only be possible for some discrete values of $\hat{c}^{2}$. We were able to derive such models in the framework of compactified heterotic M-theory for the values $\hat{c}^{2}=6,7,10$. They show an unconventional interpretation in the geometrical picture. For the hierarchy of the mass scales, the case of $\hat{c}^{2}=6$, for example, appears as a model with 2 flat extra dimensions. However, the corresponding KK-spectrum resembles that of a model with 1.6 extra dimensions. This unconventional behaviour strongly influences the couplings of the KK-modes and might have interesting applications for the role of axions in heterotic M-theory compactifications [70]. For consistent models within the framework of heterotic M-theory we can derive a lower bound: $\hat{c}^{2} \geq 6$. This bound appears as a consequence of the presence of the universal hypermultiplet in the theory compactified to 5 dimensions.

Previously discussed models, such as RS and LD, violate this bound. In a 5Dsupergravity description they require the presence of vector multiplets (but the absence of 


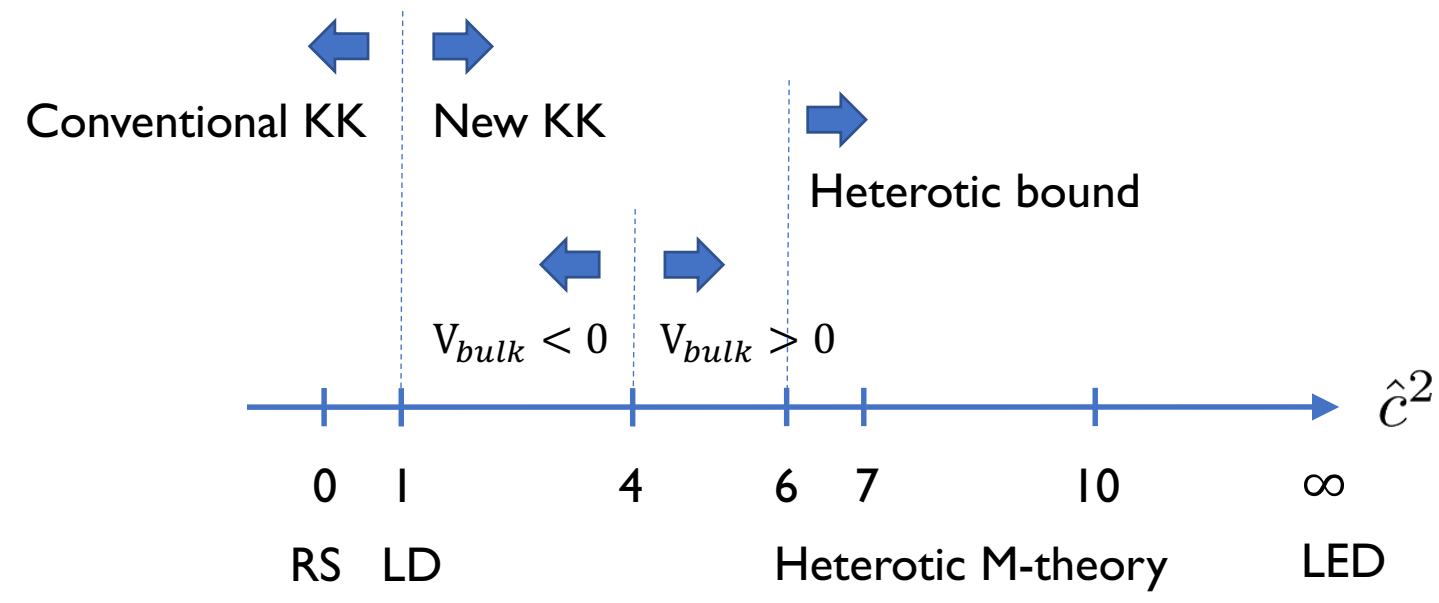

Figure 3. Critical points of $\hat{c}^{2}$. Heterotic M-theory can realize the GLD clockworks with $\hat{c}^{2} \geq 6$.

the universal hypermultiplet). It remains to be seen whether this allows a consistent uplift to a higher dimensional string- or M-theory. Models outside the heterotic M-theory bound $\hat{c}^{2} \geq 6$ do apparently require a different UV-completion. For the linear dilaton model (LD) such a completion has been suggested in the framework of a $6 \mathrm{D}$ non-critical and non-local string-like theory known as "Little String Theory". For a recent discussion and references we refer the reader to section 2.2 of [58]. It remains an open problem to understand a possible relation between these different attempts for a UV-completion. Other open questions concern the complete classification of the models that allow a consistent UV-completion. Up to now we were only able to find solutions for certain discrete values of $\hat{c}^{2}$.

The unconventional properties of the KK-spectrum and the couplings of the various KK-excitations on the various branes deserve further investigations which we will discuss in a future publication [70]. They might have relevance for researches at colliders [58] as well as axionic couplings within the framework of heterotic M-theory in view of a solution of the strong CP-problem.

\section{Acknowledgments}

This work was supported by the German Science Foundation (DFG) within the SFBTransregio TR33 "The Dark Universe". SHI acknowledges support from the National Research Foundation of Korea (NRF) grant funded by the Korea government (MSIP) (NRF2018R1C1B6006061). SHI also thanks the CERN-Korea TH Institute, where valuable comments were given to this work. HPN thanks Dieter Lüst and the ASC-Center at LMU Munich for hospitality and support. MO acknowledges partial support from National Science Centre, Poland, grants DEC-2015/18/M/ST2/00054 and DEC-2016/23/G/ST2/04301.

Open Access. This article is distributed under the terms of the Creative Commons Attribution License (CC-BY 4.0), which permits any use, distribution and reproduction in any medium, provided the original author(s) and source are credited. 


\section{References}

[1] N. Arkani-Hamed, S. Dimopoulos and G.R. Dvali, The Hierarchy problem and new dimensions at a millimeter, Phys. Lett. B 429 (1998) 263 [hep-ph/9803315] [INSPIRE].

[2] L. Randall and R. Sundrum, A Large mass hierarchy from a small extra dimension, Phys. Rev. Lett. 83 (1999) 3370 [hep-ph/9905221] [INSPIRE].

[3] I. Antoniadis, S. Dimopoulos and A. Giveon, Little string theory at a TeV, JHEP 05 (2001) 055 [hep-th/0103033] [INSPIRE].

[4] K. Choi, S.H. Im and C.S. Shin, General Continuum Clockwork, JHEP 07 (2018) 113 [arXiv: 1711.06228] [INSPIRE].

[5] P. Hořava and E. Witten, Eleven-dimensional supergravity on a manifold with boundary, Nucl. Phys. B 475 (1996) 94 [hep-th/9603142] [INSPIRE].

[6] J.E. Kim, H.P. Nilles and M. Peloso, Completing natural inflation, JCAP 01 (2005) 005 [hep-ph/0409138] [INSPIRE].

[7] R. Kappl, S. Krippendorf and H.P. Nilles, Aligned Natural Inflation: Monodromies of two Axions, Phys. Lett. B 737 (2014) 124 [arXiv:1404.7127] [InSPIRE].

[8] K. Choi, H. Kim and S. Yun, Natural inflation with multiple sub-Planckian axions, Phys. Rev. D 90 (2014) 023545 [arXiv:1404.6209] [INSPIRE].

[9] T. Higaki, K.S. Jeong, N. Kitajima and F. Takahashi, The QCD Axion from Aligned Axions and Diphoton Excess, Phys. Lett. B 755 (2016) 13 [arXiv:1512.05295] [INSPIRE].

[10] P.W. Graham, D.E. Kaplan and S. Rajendran, Cosmological Relaxation of the Electroweak Scale, Phys. Rev. Lett. 115 (2015) 221801 [arXiv:1504.07551] [INSPIRE].

[11] K. Choi and S.H. Im, Realizing the relaxion from multiple axions and its UV completion with high scale supersymmetry, JHEP 01 (2016) 149 [arXiv: 1511.00132] [INSPIRE].

[12] D.E. Kaplan and R. Rattazzi, Large field excursions and approximate discrete symmetries from a clockwork axion, Phys. Rev. D 93 (2016) 085007 [arXiv:1511.01827] [INSPIRE].

[13] N. Arkani-Hamed, A.G. Cohen and H. Georgi, (De)constructing dimensions, Phys. Rev. Lett. 86 (2001) 4757 [hep-th/0104005] [INSPIRE].

[14] C.T. Hill, S. Pokorski and J. Wang, Gauge Invariant Effective Lagrangian for Kaluza-Klein Modes, Phys. Rev. D 64 (2001) 105005 [hep-th/0104035] [InSPIRE].

[15] G.F. Giudice and M. McCullough, A Clockwork Theory, JHEP 02 (2017) 036 [arXiv: 1610.07962] [INSPIRE].

[16] N. Craig, I. Garcia Garcia and D. Sutherland, Disassembling the Clockwork Mechanism, JHEP 10 (2017) 018 [arXiv: 1704.07831] [INSPIRE].

[17] G.F. Giudice and M. McCullough, Comment on "Disassembling the Clockwork Mechanism", arXiv: 1705.10162 [INSPIRE].

[18] P. Saraswat, Weak gravity conjecture and effective field theory, Phys. Rev. D 95 (2017) 025013 [arXiv: 1608.06951] [INSPIRE].

[19] N. Fonseca, L. de Lima, C.S. Machado and R.D. Matheus, Large field excursions from a few site relaxion model, Phys. Rev. D 94 (2016) 015010 [arXiv: 1601.07183] [INSPIRE].

[20] A. Kehagias and A. Riotto, Clockwork Inflation, Phys. Lett. B 767 (2017) 73 [arXiv: 1611.03316] [INSPIRE]. 
[21] S.H. Im, H.P. Nilles and A. Trautner, Exploring extra dimensions through inflationary tensor modes, JHEP 03 (2018) 004 [arXiv: 1707.03830] [INSPIRE].

[22] M. Farina, D. Pappadopulo, F. Rompineve and A. Tesi, The photo-philic QCD axion, JHEP 01 (2017) 095 [arXiv: 1611.09855] [INSPIRE].

[23] A. Ahmed and B.M. Dillon, Clockwork Goldstone Bosons, Phys. Rev. D 96 (2017) 115031 [arXiv: 1612.04011] [INSPIRE].

[24] T. Hambye, D. Teresi and M.H.G. Tytgat, A Clockwork WIMP, JHEP 07 (2017) 047 [arXiv: 1612.06411] [INSPIRE].

[25] G. von Gersdorff, Natural Fermion Hierarchies from Random Yukawa Couplings, JHEP 09 (2017) 094 [arXiv: 1705.05430] [INSPIRE].

[26] R. Coy, M. Frigerio and M. Ibe, Dynamical Clockwork Axions, JHEP 10 (2017) 002 [arXiv: 1706. 04529] [INSPIRE].

[27] I. Ben-Dayan, Generalized Clockwork Theory, arXiv:1706.05308 [INSPIRE].

[28] D.K. Hong, D.H. Kim and C.S. Shin, Clockwork graviton contributions to muon g-2, Phys. Rev. D 97 (2018) 035014 [arXiv: 1706. 09376] [INSPIRE].

[29] S.C. Park and C.S. Shin, Clockwork seesaw mechanisms, Phys. Lett. B 776 (2018) 222 [arXiv: 1707.07364] [INSPIRE].

[30] H.M. Lee, Gauged U(1) clockwork theory, Phys. Lett. B 778 (2018) 79 [arXiv:1708. 03564] [INSPIRE].

[31] P. Agrawal, G. Marques-Tavares and W. Xue, Opening up the QCD axion window, JHEP 03 (2018) 049 [arXiv: 1708.05008] [InSPIRE].

[32] J. Kim and J. McDonald, Clockwork Higgs portal model for freeze-in dark matter, Phys. Rev. D 98 (2018) 023533 [arXiv: 1709.04105] [INSPIRE].

[33] P. Agrawal, J. Fan, M. Reece and L.-T. Wang, Experimental Targets for Photon Couplings of the QCD Axion, JHEP 02 (2018) 006 [arXiv: 1709.06085] [inSPIRE].

[34] K.S. Jeong and C.S. Shin, Peccei-Quinn Relaxion, JHEP 01 (2018) 121 [arXiv:1709.10025] [INSPIRE].

[35] O. Davidi, R.S. Gupta, G. Perez, D. Redigolo and A. Shalit, The Nelson-Barr Relaxion, arXiv:1711.00858 [INSPIRE].

[36] A. Ibarra, A. Kushwaha and S.K. Vempati, Clockwork for Neutrino Masses and Lepton Flavor Violation, Phys. Lett. B 780 (2018) 86 [arXiv:1711.02070] [INSPIRE].

[37] K.M. Patel, Clockwork mechanism for flavor hierarchies, Phys. Rev. D 96 (2017) 115013 [arXiv: 1711.05393] [INSPIRE].

[38] M. Dine, L. Stephenson Haskins, L. Ubaldi and D. Xu, Some Remarks on Anthropic Approaches to the Strong CP Problem, JHEP 05 (2018) 171 [arXiv:1801.03466] [INSPIRE].

[39] J.M. Cline and J.R. Espinosa, Axionic landscape for Higgs coupling near-criticality, Phys. Rev. D 97 (2018) 035025 [arXiv: 1801.03926] [INSPIRE].

[40] K. Choi, H. Kim and T. Sekiguchi, Late-Time Magnetogenesis Driven by Axionlike Particle Dark Matter and a Dark Photon, Phys. Rev. Lett. 121 (2018) 031102 [arXiv:1802.07269] [INSPIRE]. 
[41] A.J. Long, Cosmological Aspects of the Clockwork Axion, JHEP 07 (2018) 066 [arXiv: 1803.07086] [INSPIRE].

[42] G. Marques-Tavares and M. Teo, Light axions with large hadronic couplings, JHEP 05 (2018) 180 [arXiv: 1803.07575] [INSPIRE].

[43] Q. Bonnefoy, E. Dudas and S. Pokorski, Axions in a highly protected gauge symmetry model, arXiv: 1804.01112 [INSPIRE].

[44] J. Kim and J. Mcdonald, Freeze-In Dark Matter from a sub-Higgs Mass Clockwork Sector via the Higgs Portal, Phys. Rev. D 98 (2018) 123503 [arXiv: 1804.02661] [InSPIRE].

[45] F. Niedermann, A. Padilla and P.M. Saffin, Higher Order Clockwork Gravity, Phys. Rev. D 98 (2018) 104014 [arXiv: 1805.03523] [INSPIRE].

[46] O. Davidi, R.S. Gupta, G. Perez, D. Redigolo and A. Shalit, The hierarchion, a relaxion addressing the Standard Model's hierarchies, JHEP 08 (2018) 153 [arXiv:1806.08791] [INSPIRE].

[47] K. Choi, S. Lee, H. Seong and S. Yun, Gamma-ray spectral modulations induced by photon-ALP-dark photon oscillations, arXiv: 1806.09508 [INSPIRE].

[48] P. Agrawal, J. Fan and M. Reece, Clockwork Axions in Cosmology: Is Chromonatural Inflation Chrononatural?, JHEP 10 (2018) 193 [arXiv: 1806.09621] [INSPIRE].

[49] A. Goudelis, K.A. Mohan and D. Sengupta, Clockworking FIMPs, JHEP 10 (2018) 014 [arXiv: 1807.06642] [INSPIRE].

[50] R. Alonso, A. Carmona, B.M. Dillon, J.F. Kamenik, J. Martin Camalich and J. Zupan, A clockwork solution to the flavor puzzle, JHEP 10 (2018) 099 [arXiv:1807.09792] [INSPIRE].

[51] S.C. Park and C.S. Shin, Clockwork Higgs inflation, arXiv:1807.09952 [INSPIRE].

[52] A. Banerjee, S. Ghosh and T.S. Ray, Clockworked VEVs and Neutrino Mass, JHEP 11 (2018) 075 [arXiv: 1808.04010] [InSPIRE].

[53] N. Craig and I. Garcia Garcia, Rescuing Massive Photons from the Swampland, JHEP 11 (2018) 067 [arXiv: 1810.05647] [INSPIRE].

[54] R.T. Co, A. Pierce, Z. Zhang and Y. Zhao, Dark Photon Dark Matter Produced by Axion Oscillations, arXiv:1810.07196 [INSPIRE].

[55] P. Agrawal, N. Kitajima, M. Reece, T. Sekiguchi and F. Takahashi, Relic Abundance of Dark Photon Dark Matter, arXiv: 1810.07188 [INSPIRE].

[56] A. Kehagias and A. Riotto, The Clockwork Supergravity, JHEP 02 (2018) 160 [arXiv: 1710.04175] [INSPIRE].

[57] I. Antoniadis, A. Delgado, C. Markou and S. Pokorski, The effective supergravity of Little String Theory, Eur. Phys. J. C 78 (2018) 146 [arXiv:1710.05568] [INSPIRE].

[58] G.F. Giudice, Y. Kats, M. McCullough, R. Torre and A. Urbano, Clockwork/linear dilaton: structure and phenomenology, JHEP 06 (2018) 009 [arXiv:1711.08437] [INSPIRE].

[59] I. Antoniadis, A. Arvanitaki, S. Dimopoulos and A. Giveon, Phenomenology of TeV Little String Theory from Holography, Phys. Rev. Lett. 108 (2012) 081602 [arXiv:1102.4043] [INSPIRE].

[60] S. Stieberger, $(0,2)$ heterotic gauge couplings and their M-theory origin, Nucl. Phys. B 541 (1999) 109 [hep-th/9807124] [INSPIRE]. 
[61] E. Witten, Strong coupling expansion of Calabi-Yau compactification, Nucl. Phys. B 471 (1996) 135 [hep-th/9602070] [INSPIRE].

[62] H.P. Nilles, M. Olechowski and M. Yamaguchi, Supersymmetry breaking and soft terms in M-theory, Phys. Lett. B 415 (1997) 24 [hep-th/9707143] [INSPIRE].

[63] H.P. Nilles, M. Olechowski and M. Yamaguchi, Supersymmetry breakdown at a hidden wall, Nucl. Phys. B 530 (1998) 43 [hep-th/9801030] [INSPIRE].

[64] A. Lukas, B.A. Ovrut and D. Waldram, On the four-dimensional effective action of strongly coupled heterotic string theory, Nucl. Phys. B 532 (1998) 43 [hep-th/9710208] [InSPIRE].

[65] A. Lukas, B.A. Ovrut, K.S. Stelle and D. Waldram, The Universe as a domain wall, Phys. Rev. D 59 (1999) 086001 [hep-th/9803235] [INSPIRE].

[66] A. Kehagias, Exponential and power law hierarchies from supergravity, Phys. Lett. B 469 (1999) 123 [hep-th/9906204] [INSPIRE].

[67] D. Teresi, Clockwork without supersymmetry, Phys. Lett. B 783 (2018) 1 [arXiv: 1802.01591] [INSPIRE].

[68] A. Lukas, B.A. Ovrut, K.S. Stelle and D. Waldram, Heterotic M-theory in five-dimensions, Nucl. Phys. B 552 (1999) 246 [hep-th/9806051] [INSPIRE].

[69] K. Behrndt and S. Gukov, Domain walls and superpotentials from M-theory on Calabi-Yau three folds, Nucl. Phys. B 580 (2000) 225 [hep-th/0001082] [INSPIRE].

[70] S.H. Im, H.P. Nilles and M. Olechowski, Axion Clockworks from heterotic M-theory, in preparation. 